\title{
A WAVELET-BASED ALMOST-SURE UNIFORM APPROXIMATION OF FRACTIONAL BROWNIAN MOTION WITH A PARALLEL ALGORITHM
}

\author{
DAWEI HONG, ${ }^{* *}$ Rutgers University \\ SHUSHUANG MAN, ${ }^{* * *}$ Southwest Minnesota State University \\ JEAN-CAMILLE BIRGET* AND \\ DESMOND S. LUN, ${ }^{*}$ Rutgers University
}

\begin{abstract}
We construct a wavelet-based almost-sure uniform approximation of fractional Brownian motion (FBM) $\left(B_{t}^{(H)}\right)_{t \in[0,1]}$ of Hurst index $H \in(0,1)$. Our results show that, by Haar wavelets which merely have one vanishing moment, an almost-sure uniform expansion of FBM for $H \in(0,1)$ can be established. The convergence rate of our approximation is derived. We also describe a parallel algorithm that generates sample paths of an FBM efficiently.
\end{abstract}

Keywords: Fractional Brownian motion; wavelet expansion of stochastic integral; almostsure uniform approximation

2010 Mathematics Subject Classification: Primary 60G22

Secondary 65T60; 65Y05

\section{Introduction}

A fractional Brownian motion (FBM) $\left(B_{t}^{(H)}\right)_{t \in[0, T]}$ of Hurst index $H \in(0,1)$ is a centered Gaussian process with covariance $\mathbb{E}\left[B_{t_{1}}^{(H)} B_{t_{2}}^{(H)}\right]=\frac{1}{2}\left(t_{1}^{2 H}+t_{2}^{2 H}-\left|t_{1}-t_{2}\right|^{2 H}\right)$ for all $t_{1}, t_{2} \in$ $[0, T]$. A standard Brownian motion $(\mathrm{BM})\left(B_{t}\right)_{t \in[0, T]}$ is the special case $H=\frac{1}{2}$. There are a great number of applications of FBM in engineering and the sciences; see [4] and the references therein. The study of approximations of FBM has been active since the 1970s. A major focus is to find approximations of FBM that converge in law; see, for example, [3], [6], [7], [14], and [17], and references therein. However, practical implementations often require almostsure uniform, also termed strong uniform, approximations of FBM, which work as follows. Let $\left(B_{t}^{(H)}\right)_{t \in[0,1]}$ be an FBM of some $H \in(0,1)$. Then, with respect to the probability space where $\left(B_{t}^{(H)}\right)_{t \in[0,1]}$ is defined, the following event occurs with probability 1 . For a sample path of $\left(B_{t}^{(H)}\right)_{t \in[0,1]}$, there is a sequence of functions of $t \in[0,1]$ produced by the approximation which uniformly converges to the sample path; conversely, a sequence of functions of $t \in[0,1]$ produced by the approximation uniformly converges to a sample path of $\left(B_{t}^{(H)}\right)_{t \in[0,1]}$.

Meyer et al. [16] obtained several wavelet series expansions of FBM for $H \in(0,1)$ that almost surely and uniformly converge. Their results brought deep insights into the spectral properties of FBM. For instance, the wavelet series expansion of FBM in [16, Section 7]

Received 5 April 2012; revision received 19 May 2013.

* Postal address: Center for Computational and Integrative Biology, Department of Computer Science, Rutgers University, Camden, NJ 08102, USA.

** Email address: dhong@ camden.rutgers.edu

*** Postal address: Department of Mathematics and Computer Science, Southwest Minnesota State University, Marshall, MN 56258, USA. 
yielded a very, if not the most, efficient mathematical representation of the spectral properties of FBM - a subject that has attracted much research for decades—see [16, Section 8].

Kühn and Linde [12] showed that the optimal convergence rate that a series expansion of FBM may reach is $O\left(N^{-H} \sqrt{\log N}\right)$ if the expansion converges almost surely and uniformly. Ayache and Taqqu [2] proved that, under certain conditions, the wavelet series expansions of FBM in [16] converge at the optimal rate. Dzhaparidze and van Zanten [9] constructed a series expansion of FBM for $H \in(0,1)$ (in the frequency domain) which almost surely and uniformly converges at the optimal rate [10].

The above results will have a long-lasting impact on the study of FBM; in the meantime they stimulate further studies. Theorem 2 of Meyer et al. [16] and their Remark 4 on the theorem motivated our investigation. Haar wavelets are very convenient to compute. Moreover, the simple form of the Mandelbrot-van Ness representation of FBM [15] is likely to yield a fast algorithm. We ask whether we can construct an almost-sure uniform approximation of FBM for all $H \in(0,1)$ using the Mandelbrot-van Ness representation and Haar wavelets. In this paper we establish such an approximation of FBM for $H \in(0,1)$. Our approach is to apply Lévy's equivalence theorem (see, e.g. Theorem 9.7.1 of [8]) to a Haar wavelet-based approximation of FBM obtained from the Mandelbrot-van Ness representation, and then to carefully evaluate the wavelet coefficients.

As shown in [16], wavelet approximation of FBM is a powerful approach. A key idea of this approach is to almost surely and uniformly approximate the sample paths in a process, using independent and identically distributed (i.i.d.) Gaussian random variables with a finely designed basis of $L^{2}$ space such as Meyer's or Daubechies' wavelets. The conditions for wavelet approximations of FBM with the optimal convergence rate [2] need wavelets to have the first six vanishing moments. It is a question of whether we can use Haar wavelets that merely have the first vanishing moment to obtain an almost-sure uniform approximation of FBM for all $H$ $\in(0,1)$. We show this is possible. The convergence rate of our almost-sure uniform approximation of FBM by Haar wavelets reaches the optimal $O\left(N^{-H} \sqrt{\log N}\right)$ for $H \in\left(0, \frac{1}{2}\right]$, but the convergence slows down to rate $O\left(N^{-(1-H)} \sqrt{\log N}\right)$ for $H \in\left(\frac{1}{2}, 1\right)$ (Theorem 6.2). Haar wavelets (piecewise-constant functions) do not introduce computational errors by themselves, and our approximation (based on the Mandelbrot-van Ness representation) is in a rather simple form. These two advantages make our approximation of FBM suitable for practical applications when $H$ is not close to 1 . We also describe a parallel algorithm that efficiently generates sample paths of an FBM.

We give some preliminaries in Section 2. In Sections 3, 4, 5, and 6, we construct and prove an almost-sure uniform approximation of FBM for $H \in(0,1)$. We describe a parallel algorithm for the approximation of FBM in Section 7.

\section{Preliminaries}

Let $C_{H}=\left(\Gamma\left(H+\frac{1}{2}\right)\right)^{-1}$, the reciprocal of the gamma function at $H+\frac{1}{2}$. The Mandelbrotvan Ness stochastic integral representation of FBM [15] is

$$
B_{t}^{(H)}=C_{H} \int_{-\infty}^{t}\left((t-s)_{+}^{H-1 / 2}-(-s)_{+}^{H-1 / 2}\right) \mathrm{d} B_{s}
$$

for $H \in\left(0, \frac{1}{2}\right) \cup\left(\frac{1}{2}, 1\right)$; when $H=\frac{1}{2}$, FBM becomes BM. In what follows, we denote the underlying probability space for the above representation of FBM by $(\Omega, \mathcal{F}, \mathbb{P})$, where $\mathcal{F}$ is a standard Brownian filtration. Our construction of an almost-sure uniform approximation of 
FBM is based on a rewriting of the Mandelbrot-van Ness stochastic integral representation:

$$
B_{t}^{(H)}=I_{1}(t, H)+I_{2}(t, H)+I_{3}(t, H), \quad t \in[0,1] .
$$

Here

$$
\begin{aligned}
& I_{1}(t, H)=C_{H} \int_{0}^{t}(t-s)^{H-1 / 2} \mathrm{~d} B_{s}, \\
& I_{2}(t, H)=C_{H} \int_{-1}^{0}\left((t-s)^{H-1 / 2}-(-s)^{H-1 / 2}\right) \mathrm{d} B_{s}, \\
& I_{3}(t, H)=C_{H} \int_{-\infty}^{-1}\left((t-s)^{H-1 / 2}-(-s)^{H-1 / 2}\right) \mathrm{d} B_{s} .
\end{aligned}
$$

Let $\left(\phi_{n}\right)_{n \geq 0}$ be a complete orthonormal basis for $L^{2}[a, b]$. For $f \in L^{2}[a, b]$, we have $f=\sum_{n=0}^{\infty}\left\langle f, \phi_{n}\right\rangle \phi_{n}$ in $L^{2}[a, b]$. We take the Wiener integral on both sides of $f=$ $\sum_{n=0}^{\infty}\left\langle f, \phi_{n}\right\rangle \phi_{n}$. Then we informally interchange the order of integration and summation on the right-hand side, with $\int_{a}^{b} f(s) \mathrm{d} B_{s}=\sum_{n=0}^{\infty}\left\langle f, \phi_{n}\right\rangle \int_{a}^{b} \phi_{n}(s) \mathrm{d} B_{s}$. By Lévy's equivalence theorem we have the following result.

Theorem 2.1. It holds that $\lim _{N \rightarrow \infty} \sum_{n=0}^{N}\left\langle f, \phi_{n}\right\rangle \int_{a}^{b} \phi_{n}(s) \mathrm{d} B_{s}=\int_{a}^{b} f(s) \mathrm{d} B_{s}$ almost surely.

The Haar wavelet on $[0,1]$ is defined as follows. Let $\mathscr{H}(s)=1$ if $s \in\left[0, \frac{1}{2}\right), \mathscr{H}(s)=-1$ if $s \in\left[\frac{1}{2}, 1\right]$, and $\mathscr{H}(s)=0$ otherwise. For $n=2^{j}+k$ with $j \geq 0$ and $0 \leq k<2^{j}$, define $\mathscr{H}_{n}(s)=2^{j / 2} \mathscr{H}\left(2^{j} s-k\right)$ and $\mathscr{H}_{0}(s)=1$. The sequence $\left(\mathscr{H}_{n}\right)_{n \geq 0}$ is the Haar wavelet on [0,1], which constitutes a complete orthonormal basis for $L^{2}[0,1]$. In a similar way, we can define the Haar wavelet on any given interval $[a, b] \subset \mathbb{R}$ to constitute a complete orthonormal basis for $L^{2}[a, b]$ (see [5]).

\section{Approximation of $I_{1}(t, H)$}

We construct and prove an almost-sure uniform approximation of $I_{1}(t, H)$. Consider a family of functions $f_{t}^{(1)} \in L^{2}[0,1]$ with a parameter $t \in(0,1] \cap \mathbb{Q}$ :

$$
f_{t}^{(1)}(s)= \begin{cases}(t-s)^{H-1 / 2} & \text { if } s \in[0, t) \\ 0 & \text { otherwise. }\end{cases}
$$

By Theorem 2.1 we have

$$
\mathbb{P}\left\{\left(\int_{0}^{1} f_{t}^{(1)}(s) \mathrm{d} B_{s}\right)(\omega)=\left(\sum_{n=0}^{\infty}\left\langle f_{t}^{(1)}, \mathscr{H}_{n}\right\rangle \int_{0}^{1} \mathscr{H}_{n}(s) \mathrm{d} B_{s}\right)(\omega)\right\}=1
$$

for each $t \in(0,1] \cap \mathbb{Q}$, and, as a consequence,

$$
\mathbb{P} \bigcap_{t \in(0,1] \cap \mathbb{Q}}\left\{\left(\int_{0}^{1} f_{t}^{(1)}(s) \mathrm{d} B_{s}\right)(\omega)=\left(\sum_{n=0}^{\infty}\left\langle f_{t}^{(1)}, \mathscr{H}_{n}\right\rangle \int_{0}^{1} \mathscr{H}_{n}(s) \mathrm{d} B_{s}\right)(\omega)\right\}=1 .
$$

We define, for all $N \geq 1$,

$$
W_{1}(t, H, N)= \begin{cases}C_{H} \sum_{n=0}^{N}\left\langle f_{t}^{(1)}, \mathscr{H}_{n}\right\rangle \mathcal{L}_{n}^{(1)} & \text { for } t \in(0,1] \cap \mathbb{Q}, \\ 0 & \text { for } t=0\end{cases}
$$


Here $\mathcal{L}_{n}^{(1)}=\int_{0}^{1} \mathscr{H}_{n}(s) \mathrm{d} B_{s}, n=0,1, \ldots, N$, are i.i.d. Gaussian random variables with mean 0 and variance 1 .

In what follows, $n \in \mathbb{Z}^{+}$is said to be at level $j$ if $n=2^{j}+k$ with $j \geq 0$ and $0 \leq k<2^{j}$, and the interval $\left[k / 2^{j},(k+1) / 2^{j}\right)$ is meant to be $\left[k / 2^{j},(k+1) / 2^{j}\right]$ when $(k+1) / 2^{j}=1$.

Lemma 3.1. There is an absolute constant $D_{1}>0$ such that, for every $t \in(0,1] \cap \mathbb{Q}$ and all $N>1, \sum_{n=N+1}^{\infty}\left\langle f_{t}^{(1)}, \mathscr{H}_{n}\right\rangle^{2} \leq D_{1}\left(H(1-H) N^{2 H}\right)^{-1}$.

Proof. For $t \in(0,1] \cap \mathbb{Q}$, at each level $j=0,1, \ldots$, we partition the set

$$
\left\{n=2^{j}+k: k=0,1, \ldots, 2^{j}-1\right\}
$$

into three subsets: $g_{1}(j, t)$ consisting of all $n\left(=2^{j}+k\right)$ such that $\left[k / 2^{j},(k+1) / 2^{j}\right) \subseteq[0, t)$; $g_{2}(j, t)$ consisting of the one $n$ such that $t \in\left[k / 2^{j},(k+1) / 2^{j}\right)$; and $g_{3}(j, t)$ consisting of all $n$ such that $t \notin \bigcup_{k^{*}=k}^{2^{j}-1}\left[k^{*} / 2^{j},\left(k^{*}+1\right) / 2^{j}\right)$.

Consider a fixed $j$. By the definition of $f_{t}^{(1)}$ we have

$$
\left\langle f_{t}^{(1)}, \mathscr{H}_{n}\right\rangle=0 \quad \text { for every } n \in \mathcal{G}_{3}(j, t) .
$$

For the only $n \in \mathcal{G}_{2}(j, t)$, we denote by $\widehat{k_{t, j}}$ the $k$ that appears in $n=2^{j}+k$. We have

$$
\left\langle f_{t}^{(1)}, \mathscr{H}_{n}\right\rangle=2^{j / 2}\left[\int_{2 \widehat{k_{t, j}} / 2^{j+1}}^{\left(2 \widehat{k_{t, j}}+1\right) / 2^{j+1}} f_{t}^{(1)}(s) \mathrm{d} s-\int_{\left(2 \widehat{k_{t, j}}+1\right) / 2^{j+1}}^{\left(2 \widehat{k_{t, j}}+2\right) / 2^{j+1}} f_{t}^{(1)}(s) \mathrm{d} s\right]
$$

which implies that

$$
\begin{aligned}
\left|\left\langle f_{t}^{(1)}, \mathscr{H}_{n}\right\rangle\right| \leq 2^{j / 2} \max \{ & \int_{2 \widehat{k_{t, j}} / 2^{j+1}}^{\left(2 \widehat{k_{t, j}}+1\right) / 2^{j+1}}\left(\frac{2 \widehat{k_{t, j}}+1}{2^{j+1}}-s\right)^{H-1 / 2} \mathrm{~d} s, \\
& \left.\int_{\left(2 \widehat{k_{t, j}}+1\right) / 2^{j+1}}^{\left(2 \widehat{k_{t, j}}+2\right) / 2^{j+1}}\left(\frac{2 \widehat{k_{t, j}}+2}{2^{j+1}}-s\right)^{H-1 / 2} \mathrm{~d} s\right\} .
\end{aligned}
$$

Using this inequality, by calculation we have, for $n \in g_{2}(j, t)$,

$$
\left\langle f_{t}^{(1)}, \mathscr{H}_{n}\right\rangle^{2} \leq 2^{-2 j H}\left(2^{-(2 H+1)}\left(H+\frac{1}{2}\right)^{-2}\right) .
$$

For each $n\left(=2^{j}+k\right) \in g_{1}(j, t)$, we have

$$
\begin{aligned}
&\left\langle f_{t}^{(1)}, \mathscr{H}_{n}\right\rangle=2^{j / 2}\left[\int_{2 k / 2^{j+1}}^{(2 k+1) / 2^{j+1}}(t-s)^{H-1 / 2} \mathrm{~d} s-\int_{(2 k+1) / 2^{j+1}}^{(2 k+2) / 2^{j+1}}(t-s)^{H-1 / 2} \mathrm{~d} s\right] \\
&=\frac{2^{j / 2}}{H+1 / 2}[\left(\left(t-\frac{2 k}{2^{j+1}}\right)^{H+1 / 2}-\left(t-\frac{2 k+1}{2^{j+1}}\right)^{H+1 / 2}\right) \\
&\left.-\left(\left(t-\frac{2 k+1}{2^{j+1}}\right)^{H+1 / 2}-\left(t-\frac{2 k+2}{2^{j+1}}\right)^{H+1 / 2}\right)\right] .
\end{aligned}
$$

To facilitate our argument, we introduce a function $w$ of $h: w(h)=g\left(x_{0}+h\right)+g\left(x_{0}-h\right)-$ $2 g\left(x_{0}\right)$ where $g(\cdot)=(\cdot)^{H+1 / 2}$ and $x_{0}=t-(2 k+1) / 2^{j+1}$. We let $h=1 / 2^{j+1}$ and rewrite (3.5) as

$$
\left\langle f_{t}^{(1)}, \mathscr{H}_{n}\right\rangle=\frac{2^{j / 2}}{H+1 / 2} w(h)
$$


By Taylor's expansion,

$$
\begin{aligned}
w(h) & =w(0)+\frac{w^{\prime}(0)}{1 !} h+\frac{w^{\prime \prime}(\theta h)}{2 !} h^{2} \quad(\text { for some } 0<\theta<1) \\
& =\frac{w^{\prime \prime}(\theta h)}{2 !} h^{2} \quad\left(\text { since } w(0)=w^{\prime}(0)=0\right)
\end{aligned}
$$

Hence, we have

$$
\begin{aligned}
w(h)= & h^{2} \frac{w^{\prime \prime}(\theta h)}{2 !} \\
= & 2^{-2(j+1)} \frac{(H+1 / 2)(H-1 / 2)}{2} \\
& \times\left[\left(t-\frac{2 k+1+\theta}{2^{j+1}}\right)^{H-3 / 2}+\left(t-\frac{2 k+1-\theta}{2^{j+1}}\right)^{H-3 / 2}\right] .
\end{aligned}
$$

This equality leads us to consider the case where $n\left(=2^{j}+k\right) \in g_{1}(j, t)$ with $k+2 \leq \widehat{k_{t, j}}$. In this case, by (3.6), we have

$$
\left|\left\langle f_{t}^{(1)}, \mathscr{H}_{n}\right\rangle\right| \leq 2^{j / 2} 2^{-2(j+1)}\left|H-\frac{1}{2}\right|\left(t-\frac{2 k+1+\theta}{2^{j+1}}\right)^{H-3 / 2}
$$

(since $0<\theta<1$ and $0<H<1$ ), which yields

$$
\begin{aligned}
\left|\left\langle f_{t}^{(1)}, \mathcal{H}_{n}\right\rangle\right| & \leq 2^{j / 2} 2^{-2(j+1)}\left|H-\frac{1}{2}\right|\left(\frac{2 \widehat{k_{t, j}}}{2^{j+1}}-\frac{2 k+2}{2^{j+1}}\right)^{H-3 / 2} \\
& =\frac{|H-1 / 2|}{4} 2^{-j H}\left(\widehat{k_{t, j}}-(k+1)\right)^{H-3 / 2}
\end{aligned}
$$

Thus, for $n\left(=2^{j}+k\right) \in g_{1}(j, t)$ with $k+2 \leq \widehat{k_{t, j}}$, we have

$$
\left.\left|\left\langle f_{t}^{(1)}, \mathscr{H}_{n}\right\rangle\right|^{2} \leq 2^{-2 j H} \widehat{\left(\widehat{k_{t, j}}\right.}-(k+1)\right)^{2 H-3} \frac{|H-1 / 2|^{2}}{16} .
$$

There is one and only one $\left\langle f_{t}^{(1)}, \mathscr{H}_{n}\right\rangle$ with $n \in \mathcal{G}_{1}(j, t)$ which is not included in (3.7), namely, $n=2^{j}+\widehat{k_{t, j}}-1$. However, in this case we have

$$
\left\langle f_{t}^{(1)}, \mathscr{H}_{n}\right\rangle=2^{j / 2}\left[\int_{\left(2 \widehat{k_{t, j}}-2\right) / 2^{j+1}}^{\left(2 \widehat{k_{t, j}}-1\right) / 2^{j+1}}(t-s)^{H-1 / 2} \mathrm{~d} s-\int_{\left(2 \widehat{k_{t, j}}-1\right) / 2^{j+1}}^{2 \widehat{k_{t, j}} / 2^{j+1}}(t-s)^{H-1 / 2} \mathrm{~d} s\right]
$$

and, hence,

$$
\left\langle f_{t}^{(1)}, \mathscr{H}_{n}\right\rangle^{2} \leq \frac{2^{j}}{(H+1 / 2)^{2}} 2^{-(2 H+1) j}=2^{-2 j H}\left(H+\frac{1}{2}\right)^{-2} .
$$


Now, putting (3.3), (3.4), (3.7), and (3.8) together, there is an absolute constant $D_{1}^{*}>0$ such that, at any level $j$,

$$
\begin{aligned}
\sum_{\{n \text { at level } j\}}\left|\left\langle f_{t}^{(1)}, \mathscr{H}_{n}\right\rangle\right|^{2} & \leq D_{1}^{*} 2^{-2 j H} \sum_{\ell=1}^{\infty}\left(\frac{1}{\ell}\right)^{3-2 H} \\
& =D_{1}^{*} 2^{-2 j H}\left(1+\sum_{\ell=2}^{\infty}\left(\frac{1}{\ell}\right)^{3-2 H}\right) \\
& \leq D_{1}^{*} 2^{-2 j H}\left(1+\int_{1}^{\infty} \frac{\mathrm{d} v}{v^{3-2 H}}\right)
\end{aligned}
$$

This inequality can be written as

$$
\sum_{\{n \text { at level } j\}}\left|\left\langle f_{t}^{(1)}, \mathscr{H}_{n}\right\rangle\right|^{2} \leq \frac{D_{1}^{* *}}{1-H} 2^{-2 j H},
$$

where $D_{1}^{* *}>0$ is an absolute constant. Therefore, we have

$$
\begin{aligned}
\sum_{n=N+1}^{\infty}\left\langle f_{t}^{(1)}, \mathscr{H}_{n}\right\rangle^{2} & \leq \sum_{j=\left\lfloor\log _{2} N\right\rfloor}^{\infty} \sum_{\{n \text { at level } j\}}\left|\left\langle f_{t}^{(1)}, \mathscr{H}_{n}\right\rangle\right|^{2} \\
& \leq \sum_{j=\left\lfloor\log _{2} N\right\rfloor}^{\infty} \frac{D_{1}^{* *}}{1-H} 2^{-2 j H} \\
& =\frac{D_{1}^{* *}}{1-H} 2^{-2\left\lfloor\log _{2} N\right\rfloor H} \sum_{j=0}^{\infty} 2^{-2 j H} \\
& =\frac{D_{1}^{* *}}{1-H} 2^{-2\left\lfloor\log _{2} N\right\rfloor H} \frac{1}{1-2^{-2 H}} .
\end{aligned}
$$

Lemma 3.1 now follows from this inequality and the fact that there is an absolute constant $G>0$ such that $1 /\left(1-2^{-2 H}\right) \leq G / H$ for all $H \in(0,1)$ (because $\left.\lim _{H \rightarrow 0_{+}}\left(1-2^{-2 H}\right) / H=2 \log 2\right)$.

Lemma 3.2. For any given $H \in(0,1)$ and $q \geq 2$, we have, for all $N>1$,

$$
\mathbb{P}\left\{\sup _{t \in[0,1] \cap \mathbb{Q}}\left|I_{1}(t, H)-W_{1}(t, H, N)\right| \geq \frac{C_{H} \sqrt{2 D_{1} q}}{\sqrt{H(1-H)}} \frac{\sqrt{\log N}}{N^{H}}\right\} \leq \frac{1}{\sqrt{\pi} N^{q}},
$$

where $D_{1}$ is the absolute constant used in Lemma 3.1.

Proof. By definition, $I_{1}(0, H)=0=W_{1}(0, H, N)$. So, we focus on the case in which $t \in$ $(0,1] \cap \mathbb{Q}$. By (3.2) and the consequence of (3.1), we have

$$
\mathbb{P} \bigcap_{t \in(0,1] \cap \mathbb{Q}}\left\{\left(I_{1}(t, H)-W_{1}(t, H, N)\right)(\omega)=C_{H} \sum_{n=N+1}^{\infty}\left\langle f_{t}^{(1)}, \mathcal{H}_{n}\right\rangle \int_{0}^{1} \mathcal{H}_{n}(s) \mathrm{d} B_{s}(\omega)\right\}=1 .
$$

Here $\sum_{n=N+1}^{\infty}\left\langle f_{t}^{(1)}, \mathscr{H}_{n}\right\rangle \int_{0}^{1} \mathscr{H}_{n}(s) \mathrm{d} B_{s}$ is a Gaussian random variable with mean 0 and variance $\sum_{n=N+1}^{\infty}\left\langle f_{t}^{(1)}, \mathscr{H}_{n}\right\rangle^{2}$. By $\sigma_{1}^{2}(t, H, N)$ we denote $\sum_{n=N+1}^{\infty}\left\langle f_{t}^{(1)}, \mathscr{H}_{n}\right\rangle^{2}$. For any given 
$H \in(0,1)$ and $q \geq 2$, we have

$$
\begin{aligned}
\mathbb{P}\left\{\left|\sum_{n=N+1}^{\infty} \int_{0}^{1}\left\langle f_{t}^{(1)}, \mathcal{H}_{n}\right\rangle \mathcal{H}_{n}(s) \mathrm{d} B_{S}\right| \geq \frac{\sqrt{2 D_{1} q \log N}}{N^{H} \sqrt{H(1-H)}}\right\} \\
\quad=\frac{\sqrt{2}}{\sigma_{1}(t, H, N) \sqrt{\pi}} \int_{\sqrt{2 D_{1} q \log N / N^{H} \sqrt{H(1-H)}}}^{\infty} \exp \left(-\frac{u^{2}}{2 \sigma_{1}^{2}(t, H, N)}\right) \mathrm{d} u \\
\quad=\frac{2}{\sqrt{\pi}} \int_{\sqrt{2 D_{1} q \log N} / \sqrt{2} \sigma_{1}(t, H, N) N^{H} \sqrt{H(1-H)}}^{\infty} \mathrm{e}^{-v^{2}} \mathrm{~d} v \\
\quad \leq \frac{2}{\sqrt{\pi}} \int_{\sqrt{q \log N}}^{\infty} \mathrm{e}^{-v^{2}} \mathrm{~d} v \quad(\text { by Lemma 3.1) } \\
\quad \leq \frac{1}{\sqrt{\pi}} \int_{\sqrt{q \log N}}^{\infty} 2 v \mathrm{e}^{-v^{2}} \mathrm{~d} v \quad(\text { since } \sqrt{q \log N}>1 \text { for } q \geq 2 \text { and } N>1) .
\end{aligned}
$$

Putting this and (3.9) together completes the proof.

\section{Approximation of $I_{2}(t, H)$}

Our construction and proof for an almost-sure uniform approximation of $I_{2}(t, H)$ are similar to those for $I_{1}(t, H)$ presented in the previous section. Consider the Haar wavelet $\left(\widetilde{\mathscr{H}}_{n}\right)_{n \geq 0}$ on $[-1,0]$. We consider a family of functions $f_{t}^{(2)} \in L^{2}[-1,0]$ with a parameter $t \in[0,1] \cap \mathbb{Q}$ :

$$
f_{t}^{(2)}(s)= \begin{cases}(t-s)^{H-1 / 2}-(-s)^{H-1 / 2} & \text { if } s \in[-1,0) \\ 0 & \text { otherwise }\end{cases}
$$

By Theorem 2.1 we have

$$
\mathbb{P}\left\{\left(\int_{-1}^{0} f_{t}^{(2)}(s) \mathrm{d} B_{s}\right)(\omega)=\left(\sum_{n=0}^{\infty}\left\langle f_{t}^{(2)}, \tilde{\mathscr{H}}_{n}\right\rangle \int_{-1}^{0} \tilde{\mathscr{H}}_{n}(s) \mathrm{d} B_{s}\right)(\omega)\right\}=1
$$

for each $t \in[0,1] \cap \mathbb{Q}$, and, as a consequence,

$$
\mathbb{P} \bigcap_{t \in[0,1] \cap \mathbb{Q}}\left\{\left(\int_{-1}^{0} f_{t}^{(2)}(s) \mathrm{d} B_{s}\right)(\omega)=\left(\sum_{n=0}^{\infty}\left\langle f_{t}^{(2)}, \tilde{\mathscr{H}}_{n}\right\rangle \int_{-1}^{0} \tilde{\mathscr{H}}_{n}(s) \mathrm{d} B_{s}\right)(\omega)\right\}=1 .
$$

We define, for all $N \geq 1$,

$$
W_{2}(t, H, N)= \begin{cases}C_{H} \sum_{n=0}^{N}\left\langle f_{t}^{(2)}, \tilde{\mathscr{H}}_{n}\right\rangle \mathcal{L}_{n}^{(2)} & \text { for } t \in[0,1] \cap \mathbb{Q}, \\ 0 & \text { for } t=0 .\end{cases}
$$

Here $\mathcal{L}_{n}^{(2)}=\int_{-1}^{0} \tilde{\mathscr{H}}_{n}(s) \mathrm{d} B_{s}, n=0,1, \ldots, N$, are i.i.d. Gaussian random variables with mean 0 and variance 1 . Note that the sequence $\left(\mathcal{L}_{n}^{(2)}\right)_{n \geq 0}$ is independent of the sequence $\left(\mathcal{L}_{n}^{(1)}\right)_{n \geq 0}$ used in the definition of $W_{1}(t, H, N)$.

Lemma 4.1. There is an absolute constant $D_{2}>0$ such that, for every $t \in[0,1] \cap \mathbb{Q}$ and all $N>1, \sum_{n=N+1}^{\infty}\left\langle f_{t}^{(2)}, \widetilde{\mathscr{H}}_{n}\right\rangle^{2} \leq D_{2}\left(H(1-H) N^{2 H}\right)^{-1}$. 
Proof. For each $t \in[0,1] \cap \mathbb{Q}$,

$$
\sum_{n=N+1}^{\infty}\left\langle f_{t}^{(2)}, \tilde{\mathscr{H}}_{n}\right\rangle^{2} \leq 2\left(\sum_{n=N+1}^{\infty}\left\langle(t-s)^{H-1 / 2}, \tilde{\mathscr{H}}_{n}\right\rangle^{2}+\sum_{n=N+1}^{\infty}\left\langle(-s)^{H-1 / 2}, \tilde{\mathscr{H}}_{n}\right\rangle^{2}\right)
$$

By changing variables, the terms on the right-hand side of (4.3) become

$$
\left\langle(t-s)^{H-1 / 2}, \widetilde{\mathscr{H}}_{n}\right\rangle=\left\langle(t+s)^{H-1 / 2}, \mathscr{H}_{n}\right\rangle \quad \text { and }\left\langle(-s)^{H-1 / 2}, \widetilde{\mathscr{H}}_{n}\right\rangle=\left\langle s^{H-1 / 2}, \mathscr{H}_{n}\right\rangle .
$$

Below we estimate $\sum_{n=N+1}^{\infty}\left\langle(t+s)^{H-1 / 2}, \mathscr{H}_{n}\right\rangle^{2}$ and $\sum_{n=N+1}^{\infty}\left\langle s^{H-1 / 2}, \mathscr{H}_{n}\right\rangle^{2}$.

For $t \in(0,1] \cap \mathbb{Q}$, at each level $j$, we have, for each $n=2^{j}+k, k=0, \ldots, 2^{j}-1$,

$$
\begin{gathered}
\left\langle(t+s)^{H-1 / 2}, \mathcal{H}_{n}\right\rangle \\
=2^{j / 2}\left[\int_{2 k / 2^{j+1}}^{(2 k+1) / 2^{j+1}}(t+s)^{H-1 / 2} \mathrm{~d} s-\int_{(2 k+1) / 2^{j+1}}^{(2 k+2) / 2^{j+1}}(t+s)^{H-1 / 2} \mathrm{~d} s\right] \\
=\frac{2^{j / 2}}{H+1 / 2}\left[\left(\left(t+\frac{2 k+1}{2^{j+1}}\right)^{H+1 / 2}-\left(t+\frac{2 k}{2^{j+1}}\right)^{H+1 / 2}\right)\right. \\
\left.-\left(\left(t+\frac{2 k+2}{2^{j+1}}\right)^{H+1 / 2}-\left(t+\frac{2 k+1}{2^{j+1}}\right)^{H+1 / 2}\right)\right] .
\end{gathered}
$$

To facilitate our argument, we introduce a revised version of the function $w$ of $h$ used in the proof of Lemma 3.1. Since there will be no confusion, we denote this revised version by $w$ as $w(h)=2 g\left(x_{0}\right)-g\left(x_{0}+h\right)-g\left(x_{0}-h\right)$, where $g(\cdot)=(\cdot)^{H+1 / 2}$ and $x_{0}=t+(2 k+1) / 2^{j+1}$. We let $h=1 / 2^{j+1}$ and rewrite (4.4) as

$$
\left\langle(t+s)^{H-1 / 2}, \mathscr{H}_{n}\right\rangle=\frac{2^{j / 2}}{H+1 / 2} w(h) .
$$

Then, by Taylor's expansion,

$$
\begin{aligned}
w(h) & =w(0)+\frac{w^{\prime}(0)}{1 !} h+\frac{w^{\prime \prime}(\theta h)}{2 !} h^{2} \quad(\text { for some } 0<\theta<1) \\
& =\frac{w^{\prime \prime}(\theta h)}{2 !} h^{2} \quad\left(\text { since } w(0)=w^{\prime}(0)=0\right)
\end{aligned}
$$

Hence, we have

$$
\begin{aligned}
w(h)= & h^{2} \frac{w^{\prime \prime}(\theta h)}{2 !} \\
= & -2^{-2(j+1)} \frac{(H+1 / 2)(H-1 / 2)}{2} \\
& \times\left[\left(t+\frac{2 k+1+\theta}{2^{j+1}}\right)^{H-3 / 2}+\left(t+\frac{2 k+1-\theta}{2^{j+1}}\right)^{H-3 / 2}\right] .
\end{aligned}
$$

Using this equality, rewriting (4.4), and using the fact that $0<\theta<1$ and $0<H<1$, we have

$$
\left|\left\langle(t+s)^{H-1 / 2}, \mathscr{H}_{n}\right\rangle\right| \leq 2^{j / 2} 2^{-2(j+1)}\left|H-\frac{1}{2}\right|\left(t+\frac{2 k+1-\theta}{2^{j+1}}\right)^{H-3 / 2} .
$$


Now, as in the proof of Lemma 3.1, we denote by $2^{j}+\widehat{k_{t, j}}$ the unique $n$ such that $t \in$ $\left[\widehat{k_{t, j}} / 2^{j},\left(\widehat{k_{t, j}}+1\right) / 2^{j}\right)$. Then, there are two and only two cases to consider.

Case 1: $\widehat{k_{t, j}} \geq 1$. By (4.5) we have

$$
\begin{aligned}
\left|\left\langle(t+s)^{H-1 / 2}, \mathscr{H}_{n}\right\rangle\right| & \leq 2^{j / 2} 2^{-2(j+1)}\left|H-\frac{1}{2}\right|\left(\frac{2 \widehat{k_{t, j}}}{2^{j+1}}+\frac{2 k+1-\theta}{2^{j+1}}\right)^{H-3 / 2} \\
& \leq 2^{-2 j H}\left|H-\frac{1}{2}\right| 2^{-(H+1 / 2)}(k+1)^{H-3 / 2}
\end{aligned}
$$

Thus, there is an absolute constant $D_{2,1}>0$ such that

$$
\sum_{\{n \text { at level } j\}}\left\langle(t+s)^{H-1 / 2}, \mathscr{H}_{n}\right\rangle^{2} \leq D_{2,1} 2^{-2 j H} \sum_{\ell=1}^{\infty}\left(\frac{1}{\ell}\right)^{3-2 H} .
$$

Case 2: $\widehat{k_{t, j}}=0$. Using (4.4), we have

$$
\begin{aligned}
\left\langle(t+s)^{H-1 / 2}, \mathscr{H}_{2^{j}}\right\rangle= & 2^{j / 2}\left[\int_{0}^{1 / 2^{j+1}}(t+s)^{H-1 / 2} \mathrm{~d} s-\int_{1 / 2^{j+1}}^{2 / 2^{j+1}}(t+s)^{H-1 / 2} \mathrm{~d} s\right] \\
=\frac{2^{j / 2}}{H+1 / 2}\left[\left(\left(t+\frac{1}{2^{j+1}}\right)^{H+1 / 2}-t^{H+1 / 2}\right)\right. & \left.\quad-\left(\left(t+\frac{2}{2^{j+1}}\right)^{H+1 / 2}-\left(t+\frac{1}{2^{j+1}}\right)^{H+1 / 2}\right)\right],
\end{aligned}
$$

and, hence,

$$
\left|\left\langle(t+s)^{H-1 / 2}, \mathscr{H}_{2^{j}}\right\rangle\right| \leq \frac{2^{j / 2}}{H+1 / 2}\left(\frac{2}{2^{j}}\right)^{H+1 / 2} .
$$

For $n=2^{j}+k$ with $k=1, \ldots, 2^{j}-1$, by (4.5) we have

$$
\begin{aligned}
\left|\left\langle(t+s)^{H-1 / 2}, \mathcal{H}_{n}\right\rangle\right| & \leq 2^{j / 2} 2^{-2(j+1)}\left|H-\frac{1}{2}\right|\left(\frac{2 k+1-\theta}{2^{j+1}}\right)^{H-3 / 2} \\
& <\frac{2^{j / 2}}{H+1 / 2} 2^{-2(j+1)}\left(\frac{k}{2^{j}}\right)^{H-3 / 2}
\end{aligned}
$$

since $\left|H^{2}-\frac{1}{4}\right|<1$ for $H \in(0,1)$. Putting (4.7) and (4.8) together, we have

$$
\sum_{\{n \text { at level } j\}}\left\langle(t+s)^{H-1 / 2}, \mathscr{H}_{n}\right\rangle^{2} \leq D_{2,1} 2^{-2 j H} \sum_{\ell=1}^{\infty}\left(\frac{1}{\ell}\right)^{3-2 H} .
$$

Without loss of generality, we can let $D_{2,1}$ be the same absolute constant as in (4.6).

Using an argument similar to that used for $\left\langle(t+s)^{H-1 / 2}, \mathcal{H}_{n}\right\rangle$ presented above, there is an absolute constant $D_{2,2}>0$ such that

$$
\sum_{\{n \text { at level } j\}}\left\langle s^{H-1 / 2}, \mathscr{H}_{n}\right\rangle^{2} \leq D_{2,2} 2^{-2 j H} \sum_{\ell=1}^{\infty}\left(\frac{1}{\ell}\right)^{3-2 H} .
$$

Lemma 4.1 follows from putting (4.3), (4.9), and (4.10) together. 
Lemma 4.2. For any given $H \in(0,1)$ and $q \geq 2$, we have, for all $N>1$,

$$
\mathbb{P}\left\{\sup _{t \in[0,1] \cap \mathbb{Q}}\left|I_{2}(t, H)-W_{2}(t, H, N)\right| \geq \frac{C_{H} \sqrt{2 D_{2} q}}{\sqrt{H(1-H)}} \frac{\sqrt{\log N}}{N^{H}}\right\} \leq \frac{2}{\sqrt{\pi} N^{q}},
$$

where $D_{2}$ is the absolute constant used in Lemma 4.1.

Proof. By (4.2) and the consequence of (4.1), we have

$$
\mathbb{P} \bigcap_{t \in[0,1] \cap \mathbb{Q}}\left\{\left(I_{2}(t, H)-W_{2}(t, H, N)\right)(\omega)=C_{H} \sum_{n=N+1}^{\infty}\left\langle f_{t}^{(2)}, \tilde{\mathscr{H}}_{n}\right\rangle \int_{-1}^{0} \tilde{\mathscr{H}}_{n}(s) \mathrm{d} B_{S}(\omega)\right\}=1 .
$$

Here $\sum_{n=N+1}^{\infty}\left\langle f_{t}^{(2)}, \widetilde{\mathcal{H}}_{n}\right\rangle \int_{-1}^{0} \mathcal{H}_{n}(s) \mathrm{d} B_{s}$ is a Gaussian random variable with mean 0 and variance $\sum_{n=N+1}^{\infty}\left\langle f_{t}^{(2)}, \mathscr{H}_{n}\right\rangle^{2}$. The rest of this proof follows the same lines as the proof of Lemma 3.2.

\section{Approximation of $\mathrm{I}_{3}(t, H)$}

By the time inversion of BM, we define a BM $\left(\tilde{B}_{s}\right)_{s \in[-1,0]}: \tilde{B}_{s}=s B_{1 / s}$ for $s \in[-1,0)$ and $\tilde{B}_{0}=0$. Consider a family of functions $f_{u}^{(3)}(v) \in L^{2}[-1,0]$ with a parameter $u \in[-1,0]$ : $f_{u}^{(3)}(v)=1$ if $v \in(u, 0) ; f_{u}^{(3)}(v)=0$ otherwise. Let

$$
g_{n}(t, H)=\int_{-1}^{0}\left(\left(-u^{-1}\right)^{H-3 / 2}-\left(t-u^{-1}\right)^{H-3 / 2}\right) u^{-3}\left\langle f_{u}^{(3)}, \tilde{\mathscr{H}}_{n}\right\rangle \mathrm{d} u .
$$

Let $\left(\mathcal{L}_{n}^{(3)}\right)_{n \geq 0}$ be the sequence with $\mathcal{L}_{n}^{(3)}=\int_{-1}^{0} \tilde{\mathscr{H}}_{n}(s) \mathrm{d} \tilde{B}_{s}$, and let $\mathcal{L}^{*}=B_{-1}$. We define, for all $N \geq 1$,

$$
W_{3}(t, H, N)=C_{H}\left((t+1)^{H-1 / 2}-1\right) \mathcal{L}^{*}-C_{H}\left(H-\frac{1}{2}\right) \sum_{n=0}^{N} g_{n}(t, H) \mathcal{L}_{n}^{(3)} .
$$

Applying Lemma 3.2 to the case in which $H=\frac{1}{2}$ and the Haar wavelet $\left(\mathscr{H}_{n}\right)_{n \geq 0}$ on $[0,1]$ is replaced by its counterpart $\left(\widetilde{\mathscr{H}}_{n}\right)_{n \geq 0}$ on $[-1,0]$, we have

$$
\tilde{B}_{u}=\sum_{n=0}^{\infty}\left\langle f_{u}^{(3)}, \tilde{\mathscr{H}}_{n}\right\rangle \int_{-1}^{0} \widetilde{\mathscr{H}}_{n}(v) \mathrm{d} \tilde{B}_{v}
$$

almost surely for every $u \in[-1,0] \cap \mathbb{Q}$. Part of Theorem 6.2 below for the case $H=\frac{1}{2}$ claims that Lemma 3.2 can be extended from discrete time to continuous time. The proof for that part of Theorem 6.2 does not involve $I_{3}(t, H)$ and $I_{2}(t, H)$ (see Remark 6.1). We can in this section use the same part, i.e. (5.2) can also be extended for every $u \in[-1,0]$.

Lemma 5.1. There is an absolute constant $D_{3}>0$ such that, for any given $H \in(0,1)$ and $q \geq 2$, we have, for all $N>1$,

$$
\mathbb{P}\left\{\sup _{t \in[0,1] \cap \mathbb{Q}}\left|I_{3}(t, H)-W_{3}(t, H, N)\right| \geq \frac{C_{H} D_{3} \sqrt{q \log N}}{\sqrt{H} N^{1-H}}\right\} \leq \frac{1}{\sqrt{\pi} N^{q}} .
$$


Proof. Using stochastic integration by parts and the inversion law of BM, Garzón et al. [11] showed a technical lemma (see Lemma 3.1 therein). By this technical lemma, we almost surely have, for any fixed $t \in[0,1]$,

$$
\begin{aligned}
I_{3}(t, H)= & C_{H}\left((t+1)^{H-1 / 2}-1\right) B_{-1} \\
& -C_{H}\left(H-\frac{1}{2}\right) \int_{-1}^{0}\left(\left(-u^{-1}\right)^{H-3 / 2}-\left(t-u^{-1}\right)^{H-3 / 2}\right) u^{-3} \tilde{B}_{u} \mathrm{~d} u .
\end{aligned}
$$

Using the extension of (5.2), we have, for any fixed $t \in[0,1] \cap \mathbb{Q}$, almost surely,

$$
\begin{aligned}
& \int_{-1}^{0}\left(\left(-u^{-1}\right)^{H-3 / 2}-\left(t-u^{-1}\right)^{H-3 / 2}\right) u^{-3} \tilde{B}_{u} \mathrm{~d} u \\
& \quad=\int_{-1}^{0} \sum_{n=0}^{\infty}\left(\left(-u^{-1}\right)^{H-3 / 2}-\left(t-u^{-1}\right)^{H-3 / 2}\right) u^{-3}\left\langle f_{u}^{(3)}, \tilde{\mathscr{H}}_{n}\right\rangle \int_{-1}^{0} \tilde{\mathscr{H}}_{n}(v) \mathrm{d} \tilde{B}_{v} \mathrm{~d} u .
\end{aligned}
$$

For any fixed $t \in[0,1] \cap \mathbb{Q}$, on the right-hand side of (5.4), the summation over $n$ and the integration with respect to $\mathrm{d} u$ are interchangeable. To see this, we regard the summation as a discrete version of integration. By Lévy's equivalence theorem we have, almost surely,

$$
\sum_{n=0}^{\infty}\left\langle f_{u}^{(3)}, \tilde{\mathscr{H}}_{n}\right\rangle^{2}\left[\int_{-1}^{0} \tilde{\mathscr{H}}_{n}(v) \mathrm{d} \tilde{B}_{v}\right]^{2}=\int_{-1}^{0}\left(f_{u}^{(3)}(v)\right)^{2} \mathrm{~d} v\left[\int_{-1}^{0} \mathrm{~d} \tilde{B}_{v}\right]^{2}=|u|\left(\tilde{B}_{-1}\right)^{2}
$$

Furthermore, we have, for $H \in(0,1), u \in[-1,0)$, and $t \in[0,1]$,

$$
\begin{aligned}
\left|\left(-u^{-1}\right)^{H-3 / 2}-\left(t-u^{-1}\right)^{H-3 / 2}\right| & =\left|H-\frac{3}{2}\right| \int_{0}^{t}\left(s-u^{-1}\right)^{H-5 / 2} \mathrm{~d} s \\
& \leq\left|H-\frac{3}{2}\right|(-u)^{-H+5 / 2} \int_{0}^{t} \mathrm{~d} s \\
& \leq \frac{3}{2}(-u)^{-H+5 / 2} .
\end{aligned}
$$

By (5.5) and (5.6), we have, for $H \in(0,1)$,

$$
\begin{aligned}
\int_{-1}^{0}\{ & \left.\sum_{n=0}^{\infty}\left(\left(-u^{-1}\right)^{H-3 / 2}-\left(t-u^{-1}\right)^{H-3 / 2}\right)^{2} u^{-6}\left\langle f_{u}^{(3)}, \tilde{\mathscr{H}}_{n}\right\rangle^{2}\left[\int_{-1}^{0} \tilde{\mathscr{H}}_{n}(v) \mathrm{d} \tilde{B}_{v}\right]^{2}\right\}^{1 / 2} \mathrm{~d} u \\
& \leq \frac{3\left|\tilde{B}_{-1}\right|}{2} \int_{-1}^{0}(-u)^{-H} \mathrm{~d} u \\
& =\frac{3\left|\tilde{B}_{-1}\right|}{2(1-H)} \\
& <\infty \quad \text { with probability } 1,
\end{aligned}
$$

which implies that the stochastic Fubini theorem is applicable (see, e.g. Condition (1.5) of [18]). Then it follows from (5.4) that, for any fixed $t \in[0,1] \cap \mathbb{Q}$, almost surely,

$$
\int_{-1}^{0}\left(\left(-u^{-1}\right)^{H-3 / 2}-\left(t-u^{-1}\right)^{H-3 / 2}\right) u^{-3} \tilde{B}_{u} \mathrm{~d} u=\sum_{n=0}^{\infty} g_{n}(t, H) \int_{-1}^{0} \tilde{\mathscr{H}}_{n}(v) \mathrm{d} \tilde{B}_{v} .
$$


Throughout the rest of this proof, we suppose that $H \in(0,1) \backslash\left\{\frac{1}{2}\right\}$. Consider a family of functions $f_{x}^{(4)}(s) \in L^{2}[0,1]$ with a parameter $x \in[0,1]: f_{x}^{(4)}(s)=1$ if $s \in(0, x) ; f_{x}^{(4)}(s)=0$ otherwise. Replacing $x$ by $-u$ and $\left(\widetilde{\mathscr{H}}_{n}\right)_{n \geq 0}$ by $\left(\mathscr{H}_{n}\right)_{n \geq 0}$, we have

$$
g_{n}(t, H)=\int_{0}^{1}\left(\left(t+x^{-1}\right)^{H-3 / 2}-\left(x^{-1}\right)^{H-3 / 2}\right) x^{-3}\left\langle f_{x}^{(4)}, \mathscr{H}_{n}\right\rangle \mathrm{d} x .
$$

Recall the two conventions: $n \in \mathbb{Z}^{+}$is said to be at level $j$ if $n=2^{j}+k$ with $j \geq 0$ and $0 \leq k<2^{j}$, and the interval $\left[k / 2^{j},(k+1) / 2^{j}\right)$ is meant to be $\left[k / 2^{j},(k+1) / 2^{j}\right]$ when $(k+1) / 2^{j}=1$. For $n=2^{j}+k$, let

$$
g_{j, k}(t, H)=\int_{k / 2^{j}}^{(k+1) / 2^{j}}\left(\left(t+x^{-1}\right)^{H-3 / 2}-\left(x^{-1}\right)^{H-3 / 2}\right) x^{-3}\left\langle f_{x}^{(4)}, \mathcal{H}_{n}\right\rangle \mathrm{d} x .
$$

For simplicity, let $G_{t, H}(x)=\left(\left(t+x^{-1}\right)^{H-3 / 2}-\left(x^{-1}\right)^{H-3 / 2}\right) x^{-3}$. We have

$$
\begin{aligned}
g_{j, k}(t, H)= & \int_{2 k / 2^{j+1}}^{(2 k+1) / 2^{j+1}} G_{t, H}(x) \int_{0}^{x} \mathscr{H}_{n}(y) \mathrm{d} y \mathrm{~d} x \\
& +\int_{(2 k+1) / 2^{j+1}}^{(2 k+2) / 2^{j+1}} G_{t, H}(x) \int_{0}^{x} \mathscr{H}_{n}(y) \mathrm{d} y \mathrm{~d} x \\
= & 2^{j / 2} \int_{2 k / 2^{j+1}}^{(2 k+1) / 2^{j+1}} G_{t, H}(x) x \mathrm{~d} x-2^{j / 2} \int_{(2 k+1) / 2^{j+1}}^{(2 k+2) / 2^{j+1}} G_{t, H}(x) x \mathrm{~d} x \\
& -2^{j / 2} \int_{2 k / 2^{j+1}}^{(2 k+1) / 2^{j+1}} G_{t, H}(x) \frac{2 k}{2^{j+1}} \mathrm{~d} x+2^{j / 2} \int_{(2 k+1) / 2^{j+1}}^{(2 k+2) / 2^{j+1}} G_{t, H}(x) \frac{2 k+2}{2^{j+1}} \mathrm{~d} x .
\end{aligned}
$$

For the first two terms on the right-hand side of (5.9), we have

$$
\int_{a}^{b} G_{t, H}(x) x \mathrm{~d} x=\left.\frac{1}{H-1 / 2}\left(y^{H-1 / 2}-(t+y)^{H-1 / 2}\right)\right|_{y=1 / a} ^{y=1 / b} \text { for } b, a>0 .
$$

Let

$$
\tilde{h}_{t, H, j, k}=2^{j / 2} \int_{2 k / 2^{j+1}}^{(2 k+1) / 2^{j+1}} G_{t, H}(x) x \mathrm{~d} x-2^{j / 2} \int_{(2 k+1) / 2^{j+1}}^{(2 k+2) / 2^{j+1}} G_{t, H}(x) x \mathrm{~d} x .
$$

In the $k=0$ case we have

$$
\begin{aligned}
\tilde{h}_{t, H, j, 0}=\frac{2^{j / 2}}{H-1 / 2} & {\left[\left.\left(y^{H-1 / 2}-(t+y)^{H-1 / 2}\right)\right|_{y=\infty} ^{y=2^{j+1}}-\left.\left(y^{H-1 / 2}-(t+y)^{H-1 / 2}\right)\right|_{y=2^{j+1}} ^{y=2^{j}}\right] } \\
=\frac{2^{j / 2}}{H-1 / 2}[ & 2^{(j+1)(H-1 / 2)+1}\left(1-\left(\frac{t}{2^{j+1}}+1\right)^{H-1 / 2}\right) \\
& \left.-2^{j(H-1 / 2)}\left(1-\left(\frac{t}{2^{j}}+1\right)^{H-1 / 2}\right)\right],
\end{aligned}
$$

which implies that, for $t \in[0.1]$,

$$
\left|\tilde{h}_{t, H, j, 0}\right| \leq \frac{D_{3,1}^{*}}{2^{j(1-H)}}
$$


with an absolute constant $D_{3,1}^{*}>0$. In the $k>0$ case we have

$$
\begin{aligned}
\tilde{h}_{t, H, j, k}=\frac{2^{j / 2}}{H-1 / 2}[ & \left.y^{H-1 / 2}-(t+y)^{H-1 / 2}\right)\left.\right|_{y=2^{j+1} / 2 k} ^{y=2^{j+1} /(2 k+1)} \\
& \left.-\left.\left(y^{H-1 / 2}-(t+y)^{H-1 / 2}\right)\right|_{y=2^{j+1} /(2 k+1)} ^{y=2^{j+1} /(2 k+2)}\right] \\
=\frac{2^{j H} 2^{H-1 / 2}}{H-1 / 2} & {\left[2\left(1-\left(\frac{t(2 k+1)}{2^{j+1}}+1\right)^{H-1 / 2}\right)-\left(1-\left(\frac{t 2 k}{2^{j+1}}+1\right)^{H-1 / 2}\right)\right.} \\
& \left.-\left(1-\left(\frac{t(2 k+2)}{2^{j+1}}+1\right)^{H-1 / 2}\right)\right] .
\end{aligned}
$$

For the right-hand side of (5.11), we introduce a function $\tilde{w}$ of $h: \tilde{w}(h)=2 \tilde{g}\left(x_{0}\right)-\tilde{g}\left(x_{0}+\right.$ $h)-\tilde{g}\left(x_{0}-h\right)$. Here $\tilde{g}(x)=1-\left(1+t(2 k+1+x) / 2^{j+1}\right)^{H-1 / 2}$ and $x_{0}=0$. We then have $\tilde{h}_{t, H, j, k}=\left(2^{j H} 2^{H-1 / 2} /\left(H-\frac{1}{2}\right)\right) \tilde{w}(1)$. By Taylor's expansion we have

$$
\begin{aligned}
\tilde{w}(h) & =\tilde{w}(0)+\frac{\tilde{w}^{\prime}(0)}{1 !} h+\frac{\tilde{w}^{\prime \prime}(\theta h)}{2 !} h^{2} \quad(\text { for some } 0<\theta<1) \\
& =\frac{\tilde{w}^{\prime \prime}(\theta h)}{2 !} h^{2} \quad\left(\text { since } \tilde{w}(0)=\tilde{w}^{\prime}(0)=0\right),
\end{aligned}
$$

where

$$
\begin{aligned}
\tilde{w}^{\prime \prime}(x)= & \frac{(H-1 / 2)(H-3 / 2) t^{2}}{2^{2(j+1)}} \\
& \times\left[\left(1+\frac{t(2 k+1+x)}{2^{j+1}}\right)^{H-5 / 2}+\left(1+\frac{t(2 k+1-x)}{2^{j+1}}\right)^{H-5 / 2}\right] .
\end{aligned}
$$

Hence, we have an absolute constant $D_{3,2}^{*}>0$ such that, for $n=2^{j}+k$ with $0<k<2^{j}$,

$$
\left|\tilde{h}_{t, H, j, k}\right|=\left|\frac{2^{j H} 2^{H-1 / 2}}{H-1 / 2} \tilde{w}(1)\right|=\left|\frac{2^{j H} 2^{H-1 / 2}}{H-1 / 2} \frac{\tilde{w}^{\prime \prime}(\theta)}{2 !}\right| \leq \frac{D_{3,2}^{*}}{2^{j(2-H)}|H-1 / 2|} .
$$

Using the same method, we estimate the last two terms on the right-hand side of (5.9). Let

$$
\hat{h}_{t, H, j, k}=-2^{j / 2} \int_{2 k / 2^{j+1}}^{(2 k+1) / 2^{j+1}} G_{t, H}(x) \frac{2 k}{2^{j+1}} \mathrm{~d} x+2^{j / 2} \int_{(2 k+1) / 2^{j+1}}^{(2 k+2) / 2^{j+1}} G_{t, H}(x) \frac{2 k+2}{2^{j+1}} \mathrm{~d} x .
$$

In the $k=0$ case we have $\hat{h}_{t, H, j, 0}=2^{-j / 2} \int_{1 / 2^{j+1}}^{2 / 2^{j+1}} G_{t, H}(x) \mathrm{d} x$. Then, using (5.6), we have an absolute constant $D_{3,3}^{*}>0$ such that

$$
\left|\hat{h}_{t, H, j, 0}\right| \leq \frac{3 \times 2^{-j / 2}}{2} \int_{1 / 2^{j+1}}^{2 / 2^{j+1}} x^{-H-1 / 2} \mathrm{~d} x \leq \frac{D_{3,3}^{*}}{2^{j(1-H)}}
$$


For the $k>0$ case we have, for $b>a>0$,

$$
\begin{aligned}
\int_{a}^{b} G_{t, H}(x) \mathrm{d} x= & \int_{1 / b}^{1 / a}\left((t+u)^{H-3 / 2}-u^{H-3 / 2}\right) u \mathrm{~d} u \\
= & \left(\frac{1}{a}\right)^{H+1 / 2}\left[\frac{(a t+1)^{H-1 / 2}}{H-1 / 2}-\frac{1}{H+1 / 2}-\frac{(a t+1)^{H+1 / 2}}{(H-1 / 2)(H+1 / 2)}\right] \\
& -\left(\frac{1}{b}\right)^{H+1 / 2}\left[\frac{(b t+1)^{H-1 / 2}}{H-1 / 2}-\frac{1}{H+1 / 2}-\frac{(b t+1)^{H+1 / 2}}{(H-1 / 2)(H+1 / 2)}\right]
\end{aligned}
$$

We introduce the function $\hat{w}(h)=\hat{g}\left(x_{0}+h\right)+\hat{g}\left(x_{0}-h\right)-2 \hat{g}\left(x_{0}\right)$, where $x_{0}=0$ and

$$
\begin{aligned}
\hat{g}(x)= & \frac{2 k+1+x}{2}\left(\frac{2^{j+1}}{2 k+1+x}\right)^{H+1 / 2} \\
& \times\left[\frac{\left((2 k+1+x) t / 2^{j+1}+1\right)^{H-1 / 2}}{H-1 / 2}-\frac{1}{H+1 / 2}-\frac{\left((2 k+1+x) t / 2^{j+1}+1\right)^{H+1 / 2}}{(H-1 / 2)(H+1 / 2)}\right] .
\end{aligned}
$$

We denote by $f(x)$ the factor of $\hat{g}(x)$ in square brackets. By Taylor's expansion we have, for some $0<\theta<1$,

$$
\hat{w}(h)=\hat{w}(0)+\frac{\hat{w}^{\prime}(0)}{1 !} h+\frac{\hat{w}^{\prime \prime}(\theta h)}{2 !} h^{2}=\frac{\hat{w}^{\prime \prime}(\theta h)}{2 !} h^{2}
$$

and

$$
\begin{aligned}
& \hat{w}^{\prime \prime}(x)=2^{j(H+1 / 2)+H-1 / 2} \\
& \times\left\{\left[\frac{(H-1 / 2)(H+1 / 2) f(x)}{(2 k+1+x)^{H+3 / 2}}-\frac{(2 H-1) f^{\prime}(x)}{(2 k+1+x)^{H+1 / 2}}+\frac{f^{\prime \prime}(x)}{(2 k+1+x)^{H-1 / 2}}\right]\right. \\
& \quad+\left[\frac{(H-1 / 2)(H+1 / 2) f(-x)}{(2 k+1-x)^{H+3 / 2}}-\frac{(2 H-1) f^{\prime}(-x)}{(2 k+1-x)^{H+1 / 2}}\right. \\
&\left.\left.\quad+\frac{f^{\prime \prime}(-x)}{(2 k+1-x)^{H-1 / 2}}\right]\right\} .
\end{aligned}
$$

By (5.14) and (5.15), we have $\hat{h}_{t, H, j, k}=2^{-j / 2} \hat{w}(1)=2^{-j / 2} \hat{w}^{\prime \prime}(\theta) /(2 !)$. Then, using calculus we have an estimate as follows (specific details are available from the authors upon request). There is an absolute constant $D_{3,4}^{*}>0$ such that, for $n=2^{j}+k$ with $0<k<2^{j}$,

$$
\left|\hat{h}_{t, H, j, k}\right|=\left|\frac{2^{-j / 2} \hat{w}^{\prime \prime}(\theta)}{2 !}\right| \leq \frac{D_{3,4}^{*}}{2^{j(1-H)}}\left(\frac{1}{k+1}\right)^{H+1 / 2} .
$$

Now, putting (5.8), (5.9), (5.10), (5.12), (5.13), and (5.16) together, we have the following estimate. There is an absolute constant $D_{3,1}>0$ such that

$$
\sum_{\{n \text { at level } j\}}\left[g_{n}(t, H)\right]^{2} \leq \frac{D_{3,1}}{2^{2 j(1-H)}(H-1 / 2)^{2}} \sum_{\ell=1}^{\infty}\left(\frac{1}{\ell}\right)^{2 H+1} .
$$

Then, by (5.3), (5.4), (5.7), and (5.17), we use arguments similar to those used in the proof of Lemma 3.1 and then those used in the proof of Lemma 3.2 to complete the proof. 
Remark 5.1. In the above proof, the time inversion of $\mathrm{BM}$ adds a factor $u^{-1}$ to the integrand $\left(\left(-u^{-1}\right)^{H-3 / 2}-\left(t-u^{-1}\right)^{H-3 / 2}\right) u^{-3}$ in the second term on the right-hand side of (5.3), where the factor $u^{-2}$ in $u^{-3}$ is from a change of variable. Denote the integrand by Q. We have $\mathcal{Q} \sim u^{-H-1 / 2}$ as $u \rightarrow 0$. The exponent in $u^{-H-1 / 2}$ causes the convergence rate $O\left(N^{-(1-H)} \sqrt{\log N}\right)$ of $W_{3}(t, H)$ to $I_{3}(t, H)$. For $H \in\left(0, \frac{1}{2}\right)$, it is faster than the convergence rate $O\left(N^{-H} \sqrt{\log N}\right)$ of $W_{1}(t, H)$ to $I_{1}(t, H)$ as well as $W_{2}(t, H)$ to $I_{2}(t, H)$. But, for $H \in\left(\frac{1}{2}, 1\right)$, the convergence rate caused by the exponent becomes slow, which reflects an impact of the long-range dependence of an FBM for $H \in\left(\frac{1}{2}, 1\right)$.

\section{Approximation of FBM}

In $(\Omega, \mathcal{F}, \mathbb{P})$ we define, for $t \in[0,1] \cap \mathbb{Q}$ and $q \geq 2$,

$$
W(t, H, N)=W_{1}(t, H, N)+W_{2}(t, H, N)+W_{3}(t, H, N) .
$$

By Lemma 3.2, Lemma 4.2, Lemma 5.1, and the fact that $H>1-H$ for $H \in(1 / 2,1)$, we have the following theorem.

Theorem 6.1. There are absolute constants $C_{1,1}, C_{1,2}, C_{2,1}, C_{2,2}>0$ such that, for any given $H \in\left(0, \frac{1}{2}\right]$ and $q \geq 2$, we have, for all $N>1$,

$$
\mathbb{P}\left\{\sup _{t \in[0,1] \cap \mathbb{Q}}\left|B_{t}^{(H)}-W(t, H, N)\right| \geq \frac{C_{1,1} \sqrt{q}}{\sqrt{H(1-H)}} \frac{\sqrt{\log N}}{N^{H}}\right\} \leq \frac{C_{1,2}}{N^{q}},
$$

and, for any given $H \in\left(\frac{1}{2}, 1\right)$ and $q \geq 2$, we have, for all $N>1$,

$$
\mathbb{P}\left\{\sup _{t \in[0,1] \cap \mathbb{Q}}\left|B_{t}^{(H)}-W(t, H, N)\right| \geq \frac{C_{2,1} \sqrt{q}}{\sqrt{H(1-H)}} \frac{\sqrt{\log N}}{N^{1-H}}\right\} \leq \frac{C_{2,2}}{N^{q}} .
$$

With respect to a Hölder continuous version of an FBM, Theorem 6.1 can be extended from discrete time $t \in[0,1] \cap \mathbb{Q}$ to continuous time $t \in[0,1]$.

Theorem 6.2. An FBM $\left(B_{t}^{(H)}\right)_{t \in[0,1]}$ of $H \in(0,1)$ has a wavelet-based almost-sure uniform expansion as follows. In $(\Omega, \mathcal{F}, \mathbb{P})$ we have, for $t \in[0,1]$, with probability 1 ,

$$
\begin{aligned}
B_{t}^{(H)}= & C_{H} \sum_{n=0}^{\infty}\left\langle f_{t}^{(1)}, \mathscr{H}_{n}\right\rangle \mathcal{L}_{n}^{(1)}+C_{H} \sum_{n=0}^{\infty}\left\langle f_{t}^{(2)}, \tilde{\mathscr{H}}_{n}\right\rangle \mathcal{L}_{n}^{(2)}+C_{H}\left((t+1)^{H-1 / 2}-1\right) \mathcal{L}^{*} \\
& +C_{H}\left(H-\frac{1}{2}\right) \sum_{n=1}^{\infty} g_{n}(t, H) \mathcal{L}_{n}^{(3)},
\end{aligned}
$$

where $\left\langle f_{t}^{(1)}, \mathscr{H}_{n}\right\rangle$ and $\left(\mathcal{L}_{n}^{(1)}\right)_{n \geq 0},\left\langle f_{t}^{(2)}, \tilde{\mathscr{H}}_{n}\right\rangle$ and $\left(\mathcal{L}_{n}^{(2)}\right)_{n \geq 0}$, and $\mathcal{L}^{*}, g_{n}(t, H)$, and $\left(\mathcal{L}_{n}^{(3)}\right)_{n \geq 0}$ are the same as in (3.2), (4.2), and (5.1), respectively. Regarding ' $\sum_{n=0}^{\infty}$ ' as ' $\lim _{N \rightarrow \infty} \sum_{n=0}^{N}$,', the convergence rates are $O\left(N^{-H} \sqrt{\log N}\right)$ for $H \in\left(0, \frac{1}{2}\right]$ and $O\left(N^{-(1-H)} \sqrt{\log N}\right)$ for $H \in\left(\frac{1}{2}, 1\right)$, as expressed by (6.1) and (6.2), respectively.

Recall that by (2.1) we write $B_{t}^{(H)}$ as $I_{1}(t, H)+I_{2}(t, H)+I_{3}(t, H)$ and that these terms are then approximated by $W_{1}(t, H), W_{2}(t, H)$, and $W_{3}(t, H)$ separately. Below we provide a proof for the extension of the approximation of $I_{1}(t, H)$ by $W_{1}(t, H)$ from $t \in[0,1] \cap \mathbb{Q}$ to $t \in$ $[0,1]$ in the case $H \in\left(0, \frac{1}{2}\right]$. Proofs for $H \in\left(\frac{1}{2}, 1\right)$ and all other cases, including the extension of the approximation of $I_{2}(t, H)$ by $W_{2}(t, H)$ as well as $I_{3}(t, H)$ by $W_{3}(t, H)$, can be carried out in a similar way. 
Proof of Theorem 6.2. Using $\alpha \in \mathbb{Z}^{+}$as a parameter, let $[0,1]=\bigcup_{i=1}^{16^{\alpha}}\left[(i-1) / 16^{\alpha}\right.$, $\left.i / 16^{\alpha}\right) \cup\{1\}$. We define

$$
\begin{gathered}
\mathcal{M}_{1}(N, \alpha, i):=C_{H} \sum_{n=0}^{N}\left\langle f_{(i-1) / 16^{\alpha}}^{(1)}, \mathscr{H}_{n}\right\rangle \int_{0}^{1} \mathscr{H}_{n}(s) \mathrm{d} B_{s}, \\
Q_{1}\left(t^{*}, N, \alpha, i\right):=C_{H} \sum_{n=0}^{N}\left\langle f_{t *}^{(1)}, \mathscr{H}_{n}\right\rangle \int_{0}^{1} \mathscr{H}_{n}(s) \mathrm{d} B_{s}-\mathcal{M}_{1}(N, \alpha, i)
\end{gathered}
$$

for $t^{*} \in\left[(i-1) / 16^{\alpha}, i / 16^{\alpha}\right) \backslash \mathbb{Q}$. By Lemma 3.2 we have, for $q \geq 2$ and all $N>1$,

$$
\mathbb{P}\left\{\sup _{\alpha \in \mathbb{Z}^{+}, 1 \leq i \leq 16^{\alpha}}\left|\mathcal{M}_{1}(N, \alpha, i)-B_{(i-1) / 16^{\alpha}}^{(H)}\right| \geq \frac{C_{H} \sqrt{2 D_{1} q}}{\sqrt{H(1-H)}} \frac{\sqrt{\log N}}{N^{H}}\right\} \leq \frac{1}{\sqrt{\pi} N^{q}} .
$$

Recall the Hölder continuity of FBM. Almost surely, a sample path $B_{t}^{(H)}(\omega)(t \in[0,1])$ is Hölder continuous of order $\beta H$ for $\beta \in(0,1)$ where $\beta$ cannot be 1 by the law of the iterated logarithm; see [1]. We choose $\beta$ close to 1 , having

$$
\mathbb{P}\left\{\sup _{\alpha \in \mathbb{Z}^{+}, 1 \leq i \leq 16^{\alpha}}\left\{\left|B_{(i-1) / 16^{\alpha}}^{(H)}-B_{t^{*}}^{(H)}\right|: t^{*} \in\left[\frac{i-1}{16^{\alpha}}, \frac{i}{16^{\alpha}}\right) \backslash \mathbb{Q}\right\} \leq \frac{M}{16^{\alpha \beta}}\right\}=1,
$$

where $M>0$ is a constant depending only on the chosen $\beta$.

Note that $Q_{1}\left(t^{*}, N, \alpha, i\right)$ is a Gaussian random variable with mean 0 and variance

$$
C_{H}^{2} \sum_{n=0}^{N}\left(\int_{0}^{1}\left(f_{t *}^{(1)}(s)-f_{(i-1) / 16^{\alpha}}^{(1)}(s)\right) \mathscr{H}_{n}(s) \mathrm{d} s\right)^{2} .
$$

We estimate the variance. Without loss of generality, we suppose that $\alpha>\log _{2} N$. Then, for all $1 \leq n \leq N$ and $0 \leq k<2^{j}$, one and only one of the following three cases occurs:

$$
\begin{gathered}
{\left[\frac{i-1}{16^{\alpha}}, \frac{i}{16^{\alpha}}\right) \subset\left[\frac{2 k}{2^{j+1}}, \frac{2 k+1}{2^{j+1}}\right) ; \quad\left[\frac{i-1}{16^{\alpha}}, \frac{i}{16^{\alpha}}\right) \subset\left[\frac{2 k+1}{2^{j+1}}, \frac{2 k+2}{2^{j+1}}\right) ;} \\
{\left[\frac{i-1}{16^{\alpha}}, \frac{i}{16^{\alpha}}\right) \cap\left[\frac{k}{2^{j}}, \frac{k+1}{2^{j}}\right)=\varnothing .}
\end{gathered}
$$

Then by calculus we have the following estimate (specific details are available from the authors upon request). For any $t^{*} \in\left[(i-1) / 16^{\alpha}, i / 16^{\alpha}\right) \backslash \mathbb{Q}$,

$$
\mathbb{P}\left\{\left|Q_{1}\left(t^{*}, N, \alpha, i\right)\right|>\frac{\sqrt{2 G_{1}}}{(\sqrt{2})^{\alpha}}\right\} \leq \frac{1}{\sqrt{\pi}} \mathrm{e}^{-2^{\alpha}}
$$

with an absolute constant $G_{1}>0$. Consider the Hölder continuous version described in (6.3) over every time interval $t \in\left[(i-1) / 16^{\alpha}, i / 16^{\alpha}\right)$. Then by (6.4) we have an absolute constant $G>0$ such that

$$
\begin{gathered}
\mathbb{P} \bigcup_{i=1}^{16^{\alpha}}\left\{\sup _{t^{*} \in\left[(i-1) / 16^{\alpha}, i / 16^{\alpha}\right) \backslash \mathbb{Q}}\left|\mathcal{M}^{*}\left(t^{*}, N, \alpha, i\right)-B_{t^{*}}^{(H)}\right|\right. \\
\left.>\frac{G}{(\sqrt{2})^{\alpha}}+\frac{C_{H} \sqrt{2 D_{1} q}}{\sqrt{H(1-H)}} \frac{\sqrt{\log N}}{N^{H}}+\frac{M}{16^{\alpha \beta}}\right\} \\
\leq \frac{3 \times 16^{\alpha}}{\sqrt{\pi}} \mathrm{e}^{-2^{\alpha}}+\frac{1}{\sqrt{\pi} N^{q}} .
\end{gathered}
$$


Note that (6.5) holds for all $\alpha>\log _{2} N$. Given $H \in\left(0, \frac{1}{2}\right]$ and $q \geq 2$, by (6.5) and (6.1), we have, for all $N>1$,

$$
\begin{aligned}
& \mathbb{P}\left\{\sup _{t \in[0,1]}\left|B_{t}^{(H)}-C_{H} \sum_{n=0}^{N}\left\langle f_{t}^{(1)}, \mathscr{H}_{n}\right\rangle \mathcal{L}_{n}^{(1)}\right| \geq \frac{\sqrt{q}\left(C_{H} \sqrt{2 D_{1}}+C_{1,1}\right)}{\sqrt{H(1-H)}} \frac{\sqrt{\log N}}{N^{H}}\right\} \\
& \leq \frac{C_{1,2}+1}{\sqrt{\pi} N^{q}} .
\end{aligned}
$$

Remark 6.1. The above proof shows that $I_{1}(t, 1 / 2)$, which is a BM, has an almost sure and uniform expansion $\sum_{n=0}^{\infty}\left\langle f_{t}^{(1)}, \mathscr{H}_{n}\right\rangle \int_{0}^{1} \mathscr{H}_{n}(s) \mathrm{d} B_{s}$ for $t \in[0,1]$. The proof does not involve $I_{2}$ and $I_{3}$, which justifies our use of this expansion in the previous section.

\section{A parallel algorithm for the approximation}

We give a mathematical description of an algorithm to demonstrate how a sample path of FBM can be generated in parallel over time. Readers who are interested in parallel algorithms are referred to [13]. Theorem 6.2 implies that a sample path $B_{t}^{(H)}(\omega): t \in[0,1] \mapsto \mathbb{R}$ can almost surely and uniformly be approximated by

$$
\begin{aligned}
B_{t}^{(H)}(\omega) \approx & C_{H} \sum_{n=0}^{N}\left\langle f_{t}^{(1)}, \mathcal{H}_{n}\right\rangle \mathcal{L}_{n}^{(1)}(\omega)+C_{H} \sum_{n=0}^{N}\left\langle f_{t}^{(2)}, \widetilde{\mathscr{H}}_{n}\right\rangle \mathcal{L}_{n}^{(2)}(\omega) \\
& +C_{H}\left((t+1)^{H-1 / 2}-1\right) \mathcal{L}^{*}(\omega)+C_{H}\left(H-\frac{1}{2}\right) \sum_{n=1}^{N} g_{n}(t, H) \mathcal{L}_{n}^{(3)}(\omega) .
\end{aligned}
$$

Hence, given any time instances $t_{1}, \ldots, t_{\ell} \in[0,1]$, we can calculate approximations of $B_{t_{1}}^{(H)}(\omega), \ldots, B_{t_{\ell}}^{(H)}(\omega)$ as follows. Make $3 N+4$ independent observations of a normal distribution $\mathcal{N}(0,1)$. Denote the results from the first $N+1$ observations by $\mathcal{L}_{n}^{(1)}(\omega), n=$ $0, \ldots, N$; denote the results from the second $N+1$ observations by $\mathcal{L}_{n}^{(2)}(\omega), n=0, \ldots, N$; denote the results from the third $N+1$ observations by $\mathcal{L}_{n}^{(3)}(\omega), n=0, \ldots, N$; and denote the result from the last observation by $\mathcal{L}_{n}^{*}(\omega)$. Then, using (7.1), we compute approximations of $B_{t_{1}}^{(H)}(\omega), \ldots, B_{t_{\ell}}^{(H)}(\omega)$ separately in an arbitrarily chosen order of $t_{1}, \ldots, t_{\ell}$. This means that the $\ell$ approximations can be carried out in parallel over time $t_{1}, \ldots, t_{\ell} \in[0,1]$ on multiple (e.g., $\ell$ in the ideal case) processors available in today's computer systems.

By (7.1) we can see that the number $\ell$ of time instances is not related to $N$, the number of approximation steps. Given $N$, we can decide at what time instances $t_{1}, \ldots, t_{\ell} \in[0,1]$ we want to find approximations of $B_{t_{1}}^{(H)}(\omega), \ldots, B_{t_{\ell}}^{(H)}(\omega)$. The accuracy of such approximations is determined by $N$, as respectively shown by the deviation bounds (6.1) and (6.2) for the cases $H \in\left(0, \frac{1}{2}\right]$ and $H \in\left(\frac{1}{2}, 1\right)$. Given time instances $t_{1}, \ldots, t_{\ell} \in[0,1]$, we can choose the number $N$ of approximation steps to ensure the accuracy of the approximation by the above two deviation bounds.

By using the Mandelbrot-van Ness representation and Haar wavelets, the coefficients on the right-hand side of (7.1), i.e. $\left\langle f_{t}^{(1)}, \mathscr{H}_{n}\right\rangle,\left\langle f_{t}^{(2)}, \mathscr{H}_{n}\right\rangle$, and $g_{n}(t, H)$, are easy to compute.

\section{Acknowledgements}

The authors are grateful to the anonymous referee for instructive comments which led to significant improvements to the original version of this paper. The authors thank the editor for granting them opportunities to improve the paper. 


\section{References}

[1] Arcones, M. A. (1995). On the law of the iterated logarithm for Gaussian processes. J. Theoret. Prob. 8, 877-903.

[2] Ayache, A. AND TAqQU, M. S. (2003). Rate optimality of wavelet series approximations of fractional Brownian motion. J. Fourier Anal. Appl. 9, 451-471.

[3] Bardina, X., Jolis, M. And Tudor, C. A. (2003). Weak convergence to the fractional Brownian sheet and other two-parameter Gaussian processes. Statist. Prob. Lett. 65, 317-329.

[4] Biagini, F., Hu, Y., ØKSEndal, B. And Zhang, T. (2008). Stochastic Calculus for Fractional Brownian Motion and Applications. Springer, London.

[5] Daubechies, I. (1992). Ten Lectures on Wavelets. SIAM, Philadelphia, PA.

[6] Davydov, Yu. A. (1970). The invariance principle for stationary processes. Teor. Vero. Primen. 15, 498-509.

[7] Delgado, R. and Jolis, M. (2000). Weak approximation for a class of Gaussian process. J. Appl. Prob. 37, 400-407.

[8] Dudley, R. M. (2002). Real Analysis and Probability. Cambridge University Press.

[9] Dzhaparidze, K. and van Zanten, H. (2004). A series expansion of fractional Brownian motion. Prob. Theory Relat. Fields 130, 39-55.

[10] Dzhaparidze, K. and van Zanten, H. (2005). Optimality of an explicit series expansion of the fractional Brownian sheet. Statist. Prob. Lett. 71, 295-301.

[11] Garzón, J., Gorostiza, L. G. And León, J. A. (2009). A strong uniform approximation of fractional Brownian motion by means of transport processes. Stoch. Process. Appl. 119, 3435-3452.

[12] KüHn, T. AND Linde, W. (2002). Optimal series representation of fractional Brownian sheets. Bernoulli 8, 669-696.

[13] Leighton, F. T. (1992). Introduction to Parallel Algorithms and Architectures. Morgan Kaufmann, San Mateo, CA.

[14] LI, Y. AND DAI, H. (2011). Approximations of fractional Brownian motion. Bernoulli 17, 1195-1216.

[15] Mandelbrot, B. B. And Van Ness, J. W. (1968). Fractional Brownian motions, fractional noises and applications. SIAM Rev. 10, 422-437.

[16] Meyer, Y., Sellan, F. and Taqqu, M. S. (1999). Wavelets, generalized white noise and fractional integration: the synthesis of fractional Brownian motion. J. Fourier Anal. Appl. 5, 465-494.

[17] TAQQU, M. S. (1974/75). Weak convergence to fractional Brownian motion and to the Rosenblatt process. Z. Wahrscheinlichkeitsth. 31, 287-302.

[18] VeraAR, M. (2012). The stochastic Fubini theorem revisited. Stochastics 84, 543-551. 